\title{
Protective effect of astragalosides from Radix Astragali on adriamycin-induced podocyte injury
}

\author{
YI-PA SAI", YUAN-CHUN SONG* ${ }^{*}$ XING-XING CHEN, XUAN LUO, JING LIU and WEI-JING CUI \\ Department of Pediatrics, Gansu Province People's Hospital, Lanzhou, Gansu 730000, P.R. China
}

Received August 11, 2017; Accepted January 26, 2018

DOI: $10.3892 /$ etm.2018.5933

\begin{abstract}
Nephrotic syndrome (NS) is the most common kidney disease in clinical practice and may lead to end-stage renal failure. Astragalosides (AST) have been clinically tested for the treatment of NS, but their mechanism of action has remained to be elucidated. The aim of the present study was to investigate the effect of AST on the structure and function of podocytes with adriamycin (ADR)-induced damage and to elucidate the underlying molecular mechanisms. The mouse podocyte clone 5 (MPC5) immortalized mouse podocyte cell line was treated with $0.5 \mu \mathrm{mol} / 1 \mathrm{ADR}$ to establish a podocyte injury model. The MPC5 podocytes were divided into a control group, a podocyte injury group and a low-, medium- and high-concentration AST treatment group. The results indicated that the survival rate of the podocyte injury group was significantly decreased compared with that in the control group and each AST-treated group had an increased survival rate compared with that in the podocyte injury group. Furthermore, each dose of AST significantly inhibited the ADR-associated increases the levels of lactate dehydrogenase and malondialdehyde and the decrease in the activity of superoxide dismutase in MPC5 podocytes. In addition, AST improved the migration ability of MPC5 podocytes and suppressed the cytoskeletal rearrangement associated with ADR-induced damage. Furthermore, matrix metalloproteinase (MMP)-2 and -9 were decreased in the podocyte injury group, which was inhibited by different concentrations of AST. Thus, AST was able to maintain the balance of oxidative
\end{abstract}

Correspondence to: Miss Yi-Pa Sai, Department of Pediatrics, Gansu Province People's Hospital, 204 Donggang West Road, Lanzhou, Gansu 730000, P.R. China

E-mail: suade10@sohu.com

*Contributed equally

Abbreviations: NS, nephrotic syndrome; AST, astragalosides; ADR, adriamycin; MPC5, mouse podocyte clone 5; ASTL, low-concentration AST; ASTM, medium-concentration AST; ASTH, high-concentration AST; LDH, lactate dehydrogenase; MDA, malonaldehyde; SOD, superoxide dismutase; MMP, matrix metalloproteinase; MT1, metallothionein 1

Key words: astragalosides, podocyte, adriamycin, cytoskeleton, oxidative stress, migration, apoptosis stress in podocytes cultured with ADR and protect them from ADR-induced injury. The mechanism may be associated with the upregulation of MMPs.

\section{Introduction}

Nephrotic syndrome (NS) is one of the most common kidney diseases in children and adults. Its pathology includes proteinuria, with consequent hypoalbuminemia and generalized edema. Proteinuria is considered to be caused by impaired glomerular function. Podocytes (foot processes) represent an important structure of the glomerular filtration membrane. Podocyte injury is considered to be one of the most critical factors in the development of proteinuria caused by glomerular filtration barrier dysfunction and continuous injury may cause severe apoptosis, leading to proteinuria, glomerulosclerosis and renal impairment; the major reason is that podocytes are highly differentiated cells with specific structural and biological functions, which are not renewable after sustained injury $(1,2)$. To date, a large number of studies have indicated that podocyte injury, loss and dysfunction have an important role in pathologies including focal segmental glomerulosclerosis and membranous nephropathy (3-5). Immunosuppressants (e.g., steroids or cyclosporine A) have been used based on anecdotal evidence to treat NS (6). Unless a contraindication exists, glucocorticoids (GC) continue to be the first-line therapy (7). However, chronic exposure to GC results in significant toxicity, including obesity, growth retardation, hypertension, poor bone health and cosmetic effects, particularly for children. Therefore, novel therapeutic strategies for NS are required.

Chinese medicinal herbs are considered to be a promising source of potential treatments, as the large variety of species contains pharmaceutically active components with broad medicinal applications. Radix Astragali (Huangqi), the root of Astragalus membranaceus Bunge, is widely used in Traditional Chinese Medicine due to its anti-inflammatory, anti-oxidative, immunoregulatory and neuroprotective activities $(8,9)$, and is also commonly used in the treatment of NS (10-12). The principal bioactive components extracted from Radix Astragali are known as astragalosides (AST). The results of a previous study suggested that intravenous infusion of AST was safe and well tolerated in healthy Chinese volunteers, and the adverse events, including raised total bilirubin and rash, were mild and spontaneously resolved (13). A study by Wang et al (14) confirmed that AST improved the expression of the glomerular 
podocyte-associated proteins nephrin and podocin in rats with adriamycin (ADR)-induced nephropathy. However, the effects of AST on podocytes, their signaling transduction pathways and the internal regulatory mechanisms require further study. ADR-induced NS is a classical NS model, which was first reported by Bertani et al (15). ADR induces thinning of the glomerular endothelium and podocyte effacement associated with the loss of the size- and charge-specific barrier for the filtration of plasma proteins. Therefore, the present study used this ADM-induced podocyte injury model, which was treated with different doses of AST and oxidative stress, proliferation and migration were assessed to explore its effect on injured podocytes, as well as the underlying mechanisms.

\section{Materials and methods}

Instruments and materials. AST was purchased from Shanghai Jingdu Biotechnology Co., Ltd. (Shanghai, China). The mouse podocyte clone 5 (MPC5) cell line was obtained from Tong Pai Bio-tech Co., Ltd. (Shanghai, China). ADR was purchased from Wuhan Xinwei Ye Chemical Co., Ltd. (Wuhan, China). Hoechst 33342 staining solution and fluorescein isothiocyanate (FITC)-labeled phalloidin was obtained from Sigma-Aldrich (Merck KGaA, Darmstadt, Germany). The reagent kits for lactate dehydrogenase (LDH; cat. no. A020-1), malondialdehyde (MDA; cat.no.A003-4) and superoxide dismutase (SOD; cat.no. A001-1-1) were supplied by Nanjing Jiancheng Bioengineering Institute (Nanjing, China). The ELISA kits for matrix metalloproteinase (MMP)-2 (cat. no. ml002275889) and MMP-9 (cat. no. ml037717 were purchased from Shanghai Enzyme-linked Biotechnology Co., Ltd. (Shanghai, China). In addition, a SpectraMAX 190 microplate reader (Molecular Devices, Sunnyvale, CA, USA), a Transwell chamber (Corning Inc., Corning, NY, USA; pore size, $8 \mu \mathrm{m}$ ), an inverted microscope (Axio Observer A; Zeiss Inc., Wetzlar, Germany) and a laser-scanning confocal microscope (Olympus FluoView ${ }^{\mathrm{TM}}$ 1000; Olympus Corp., Tokyo, Japan) were used in the present study.

Cell culture and treatment. The MPC5 mouse podocytes (Tong Pai Bio-tech Co., Ltd, Shanghai, China) were recovered from freezing and maintained in RPMI 1640 medium (Gibco; Thermo Fisher Scientific, Inc., Waltham, MA, USA) containing $10 \%$ heat-inactivated fetal calf serum (HyClone; GE Healthcare, Little Chalfont, UK). The MPC5 cells were cultured and expanded by growth in a medium containing $10 \mathrm{U} / \mathrm{ml}$ interferon- $\gamma$ (Peprotech, Rocky Hill, NJ, USA) at $37^{\circ} \mathrm{C}$ with $100 \%$ relative humidity and $5 \% \mathrm{CO}_{2}$, When cells reached $70 \%$ confluence, they were subcultured and treated in groups.

Grouping. MPC5 podocytes were divided into a control group (complete medium), podocyte injury group $(0.5 \mu \mathrm{mol} / 1$ ADR), low-concentration AST treatment group (ASTL group; $0.5 \mu \mathrm{mol} / 1 \mathrm{ADR}+50 \mu \mathrm{g} / \mathrm{ml} \mathrm{AST})$, medium-concentration AST treatment group (ASTM group; $0.5 \mu \mathrm{mol} / 1 \mathrm{ADR}+100 \mu \mathrm{g} / \mathrm{ml}$ AST) and high-concentration AST treatment group (ASTH group; $0.5 \mu \mathrm{mol} / 1 \mathrm{ADR}+200 \mu \mathrm{g} / \mathrm{ml}$ AST). Each cell group was incubated at $37^{\circ} \mathrm{C}$ for $24 \mathrm{~h}$, digested with pancreatin (0.25\%; Lanzhou Lihe Biotechnology Co., Ltd., Lanzhou, China) for subculture (at $37^{\circ} \mathrm{C}$ for $24 \mathrm{~h}$ ) and used for further experimentation.
Cell proliferation assay. The differentiated and mature podocytes were seeded into 96 -well plates at a density of $2.0 \times 10^{3}$ per well. After full adherence, cells were treated as aforementioned and each experimental condition was performed in six wells. After $24 \mathrm{~h}$ of incubation, MTT reagent $(5 \mathrm{mg} / \mathrm{ml}, 20 \mu \mathrm{l})$ was added and the cells were incubated for another $4 \mathrm{~h}$. After the supernatant was discarded, $150 \mu \mathrm{l}$ dimethyl sulfoxide (DMSO) was added to each well, followed by agitation for $15 \mathrm{~min}$. The optical density of every well at $570 \mathrm{~nm}$ (OD570) was read with an ELISA plate reader and the survival rate was calculated according to the following formula: Survival rate $=\mathrm{OD} 570_{\text {treatment group }} / \mathrm{OD} 570_{\text {control group }} \times 100 \%$. The experiment was repeated three times.

Detection of hypoxic damage and oxidative stress indices. After the above mentioned treatments, cells were washed three times with PBS, followed by radioimmunoprecipitation assay buffer with repeated freezing and thawing. Subsequently, the lysates were analysed with the LDH, MDA and SOD kits following the manufacturer's instructions, and the OD was measured at 490, 532 and $550 \mathrm{~nm}$, respectively, to calculate the production or activity of LDH, MDA and SOD.

Observation of the cytoskeleton. Cells in each group were fixed with $4 \%$ paraformaldehyde at room temperature for 10 min, washed once with PBS, treated with $0.1 \%$ Triton X-100 for $10 \mathrm{~min}$ and blocked with $5 \%$ bovine serum albumin (cat. no. A8010-10g; Beijing Solarbio Science \& Technology Co., Ltd., Beijing, China) for $30 \mathrm{~min}$. After washing twice with PBS, $10 \mu \mathrm{g} / \mathrm{ml}$ FITC-phalloidin was added to the central cell surface, followed by incubation away from light at $37^{\circ} \mathrm{C}$ for $2 \mathrm{~h}$ and washing for three times with PBS for 5 min each time. Hoechst 33342 staining solution was then added, followed by incubation away from light at room temperature for $10 \mathrm{~min}$. Subsequent to washing for 3 times with PBS for 5 min each time, the cells were mounted with anti-fade fluorescence mounting medium, observed with the Olympus FluoView ${ }^{\mathrm{TM}} 1000$ laser confocal scanning microscope and images were captured.

Cell migration assay. Each group of MPC5 podocytes was pre-processed by starvation treatment for $6 \mathrm{~h}$, digested with $0.25 \%$ pancreatin (Lanzhou Lihe Biotechnology Co., Ltd., Lanzhou, China) at room temperature for $2 \mathrm{~min}$, the cell density was adjusted and $2 \times 10^{5}$ cells were inoculated to each compartment of a Transwell chamber. A total of $500 \mu \mathrm{l}$ medium containing $10 \%$ serum was added to the bottom wells of the 24-well plate and the plate was cultured at $37^{\circ} \mathrm{C}$ for $12 \mathrm{~h}$, followed by fixing with methanol at $4^{\circ} \mathrm{C}$ for $10 \mathrm{~min}$ and staining with $0.1 \%$ crystal violet for $30 \mathrm{~min}$ at room temperature. The cells were observed and images were captured under an inverted microscope after wiping off the cells in the upper chamber with cotton swabs. Subsequently, the crystal violet was extracted by rinsing each well with $33 \%$ acetic acid. The eluent was added to a 96-well plate and the absorbance was read with a microplate reader at a wavelength $570 \mathrm{~nm}$, which indirectly reflects the number of migrating cells.

ELISA. The levels of MMP-2 and MMP-9 in the supernatants of the cells were determined by ELISA. The MPC5 culture medium was collected and centrifuged at a speed of 13,000 $\mathrm{x}$ for $15 \mathrm{~min}$ 
at $4^{\circ} \mathrm{C}$ to pelletize the debris. The levels of the two proteins were determined according to the manufacturer's protocols for the kits. The colorimetric reaction was measured at $450 \mathrm{~nm}$.

Statistical analysis. The experiment was repeated at least three times and values are expressed as the mean \pm standard deviation. Statistical analyses were performed using SPSS 20.0 (IBM Corp., Armonk, NY, USA). A Student's t-test was used to compare the means between two groups and comparisons between multiple groups were made by one-way analysis of variance, after that a post-hoc Tukey's tests was conducted. $\mathrm{P}<0.05$ was considered to indicate a statistically significant difference.

\section{Results}

AST improves the survival rate of podocytes with ADR-induced injury. The results of the MTT assay indicated that the survival rate of MPC5 podocytes was $52 \pm 2.4 \%$ after treatment with $0.5 \mu \mathrm{mol} / 1 \mathrm{ADR}$ for $24 \mathrm{~h}$, which was significantly reduced compared with that in the control group $(\mathrm{P}<0.01$; Fig. 1$)$. The survival rates in the ASTL, ASTM and ASTH groups were $63 \pm 3.4,75 \pm 3.2$ and $67 \pm 3.0$, respectively, which were higher than those in the podocyte injury group $(\mathrm{P}<0.05)$. However, no dose-dependent effect was observed among the groups treated with different AST concentrations.

AST reduces the oxidative stress levels of podocytes with ADR-induced injury. Quantification of LDH released from each group of MPC5 podocytes with kits indicated that treatment of MPC5 cells with $0.5 \mu \mathrm{mol} / 1$ ADR for $24 \mathrm{~h}$ resulted in a $\sim 2$-fold increase compared with the control group $(\mathrm{P}<0.05)$. The low, medium and high concentrations of AST reduced the ADR-induced increase of $\mathrm{LDH}$ generation to $1.64 \pm 0.07-$, $1.33 \pm 0.09$ - and $1.46 \pm 0.11$-fold of that in the control group, respectively, which was significantly lower than that in the ADR injury group $(\mathrm{P}<0.05$; Table I). Furthermore, compared with that in the control group, the 24-h treatment of MPR 5 podocytes with $0.5 \mu \mathrm{mol} / 1$ ADR significantly increased the relative content of MDA in podocytes to $2.71 \pm 0.40$-fold $(\mathrm{P}<0.05)$, while decreasing the SOD activity to $0.78 \pm 0.01$-fold $(\mathrm{P}<0.05)$. The low, medium and high concentrations of AST inhibited the ADR-induced increase of relative MDA generation to $2.06 \pm 0.23,1.70 \pm 0.19$ and $1.98 \pm 0.26$, respectively, which was significantly different from that in the ADR injury group $(\mathrm{P}<0.05)$. Furthermore, AST at the low, medium and high concentrations significantly increased the SOD activities relative to those in the control group from $0.77 \pm 0.01$ in the ADR group to $0.86 \pm 0.03,0.93 \pm 0.02$ and $0.87 \pm 0.03$, respectively $(\mathrm{P}<0.05$; Table I).

AST attenuates ADR-induced decreases in the migratory capacity of podocytes. The results of the Transwell cell migration assay indicated that compared with that in the control group, the mobility of podocytes in each experimental group changed significantly. The relative mobility of podocytes in the ADR injury group was $35 \pm 2.1 \%$, of that of the normal MPC5 cells $(\mathrm{P}<0.05)$. AST at the low, medium and high concentration inhibited the ADR-induced decline of podocyte migration ability ( $\mathrm{P}<0.05$ vs. control group). However, no statistically significant difference was present between the ASTM and ASTH groups ( $\mathrm{P}>0.05$; Figs. 2 and 3 ).
Table I. Levels of LDH, MDA and SOD in podocytes in different groups.

\begin{tabular}{llll}
\hline Group & LDH & MDA & SOD \\
\hline Control & $1.00 \pm 0.00$ & $1.00 \pm 0.00$ & $1.00 \pm 0.00$ \\
Podocyte injury & $1.95 \pm 0.11^{\mathrm{a}}$ & $2.71 \pm 0.40^{\mathrm{a}}$ & $0.77 \pm 0.01^{\mathrm{a}}$ \\
ASTL & $1.64 \pm 0.07^{\mathrm{b}}$ & $2.06 \pm 0.21^{\mathrm{b}}$ & $0.86 \pm 0.03^{\mathrm{b}}$ \\
ASTM & $1.33 \pm 0.09^{\mathrm{c}}$ & $1.70 \pm 0.19^{\mathrm{c}}$ & $0.93 \pm 0.02^{\mathrm{c}}$ \\
ASTH & $1.46 \pm 0.11^{\mathrm{c}}$ & $1.96 \pm 0.21^{\mathrm{c}}$ & $0.89 \pm 0.03^{\mathrm{c}}$ \\
\hline
\end{tabular}

${ }^{\mathrm{a}} \mathrm{P}<0.05$ vs. the control group; ${ }^{\mathrm{b}} \mathrm{P}<0.05,{ }^{\mathrm{c}} \mathrm{P}<0.01$ vs. the podocyte injury group. Values are expressed as the mean \pm standard deviation. LDH, lactate dehydrogenase; MDA, malondialdehyde; SOD, superoxide dismutase; ASTL/M/H, treatment with low/medium/high concentration of astragaloside.

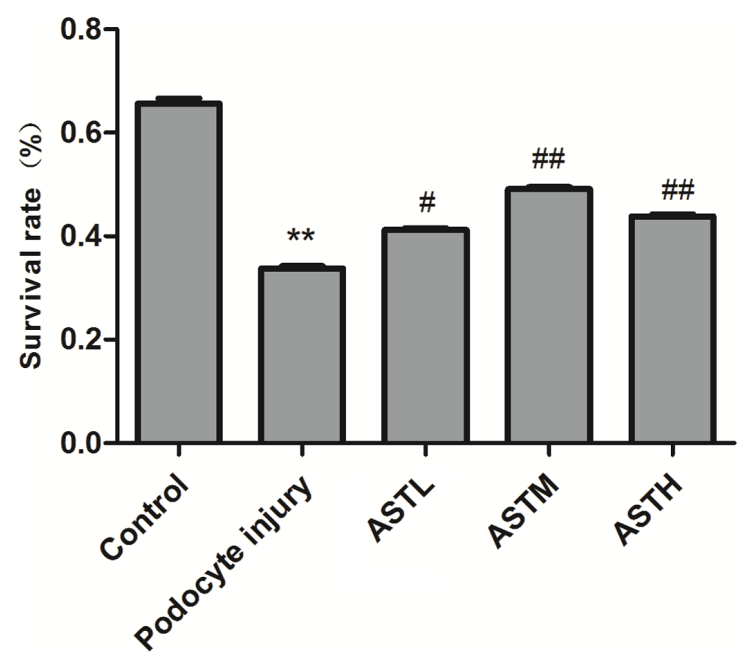

Figure 1. Comparison of podocyte survival rates between the groups. Values are expressed as the mean \pm standard deviation $(n=3) .{ }^{* *} \mathrm{P}<0.01$ vs. the control group; ${ }^{\#} \mathrm{P}<0.05,{ }^{\# \#} \mathrm{P}<0.01$ vs. the podocyte injury group. ASTL/M/H, treatment with low/medium/high concentration of astragaloside.

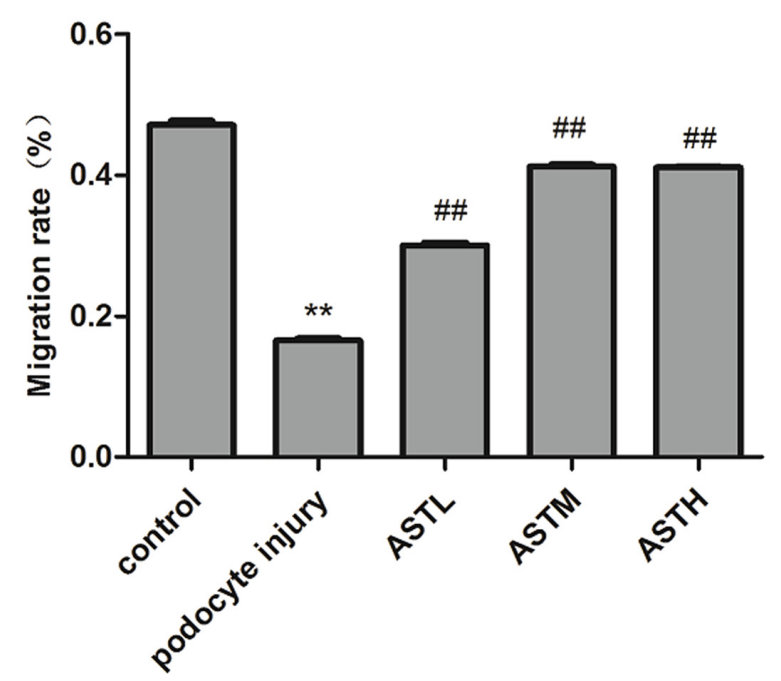

Figure 2. Comparison of the podocyte migration rate between the groups. Values are expressed as the mean \pm standard deviation $(n=3) .{ }^{* *} \mathrm{P}<0.01$ vs. the control group; ${ }^{\#} \mathrm{P}<0.01$ vs. the podocyte injury group. ASTL/M/H, treatment with low/medium/high concentration of astragaloside. 
A
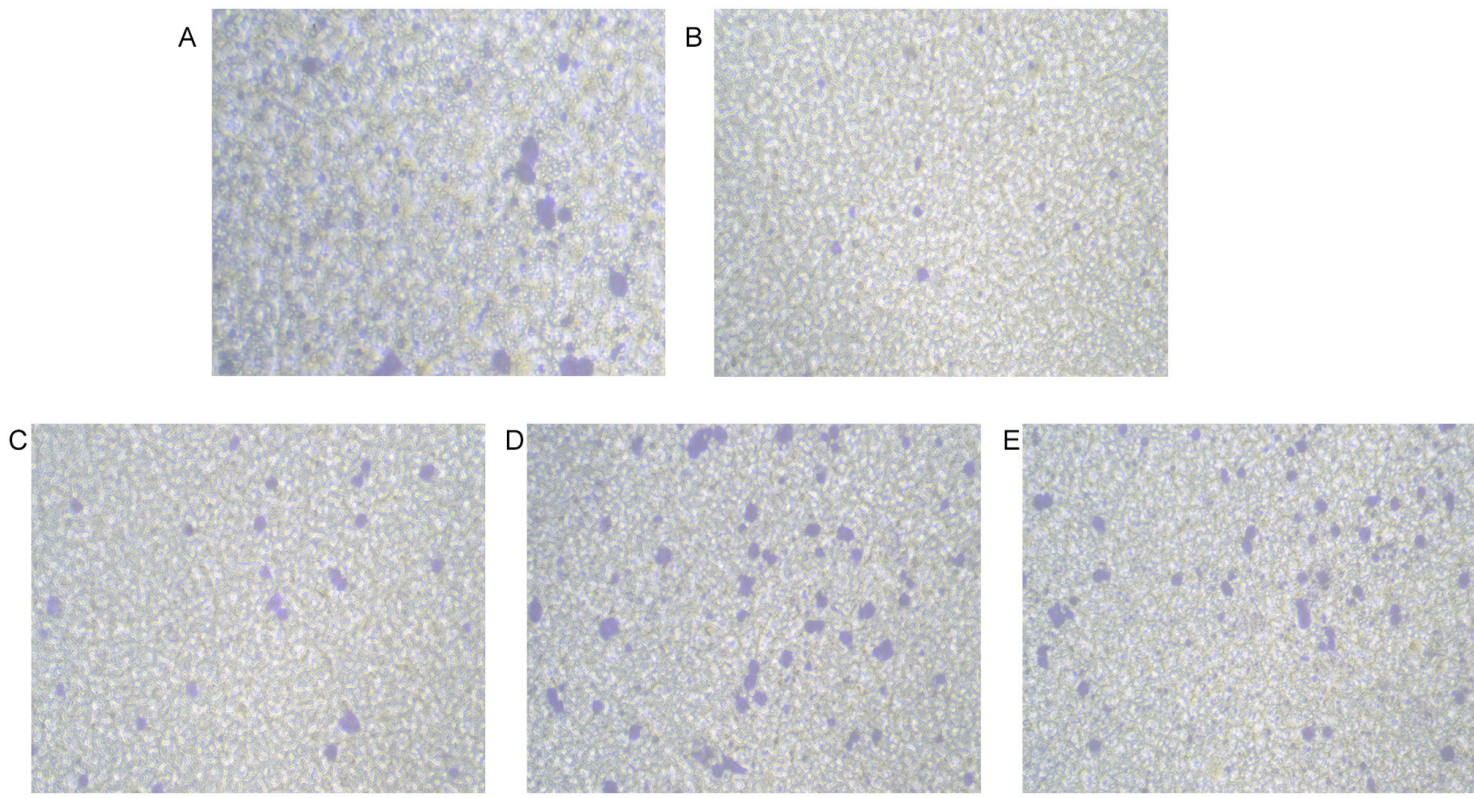

Figure 3. Migration of podocytes in different groups. Images of crystal violet-stained cells on the lower side of the Transwell assay membranes are presented (magnification, x200). (A) Control group; (B) podocyte injury group; (C) ASTL group; (D) ASTM group; (E) ASTH group. ASTL/M/H, treatment with low/medium/high concentration of astragaloside.

A
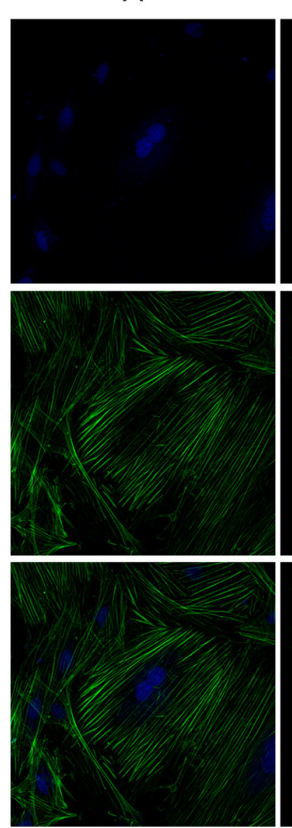

B
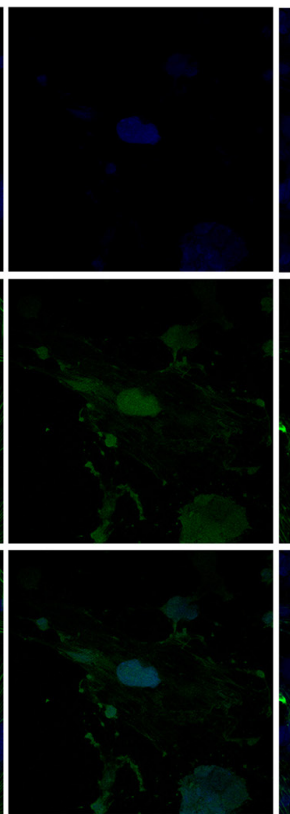

C
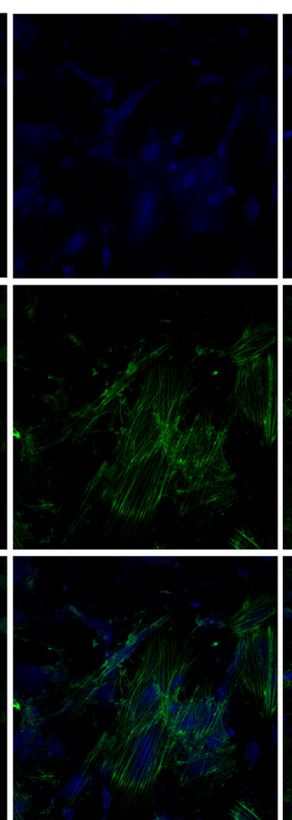

D
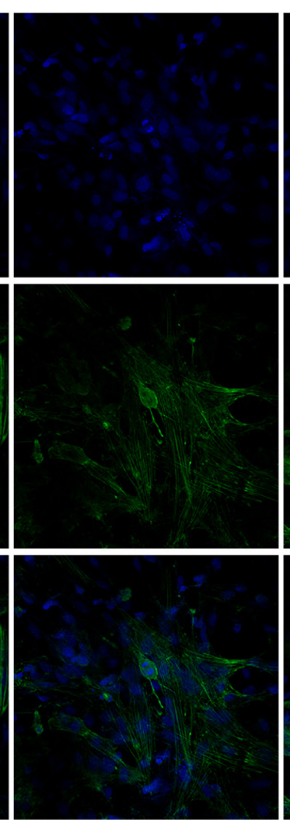

$\mathrm{E}$
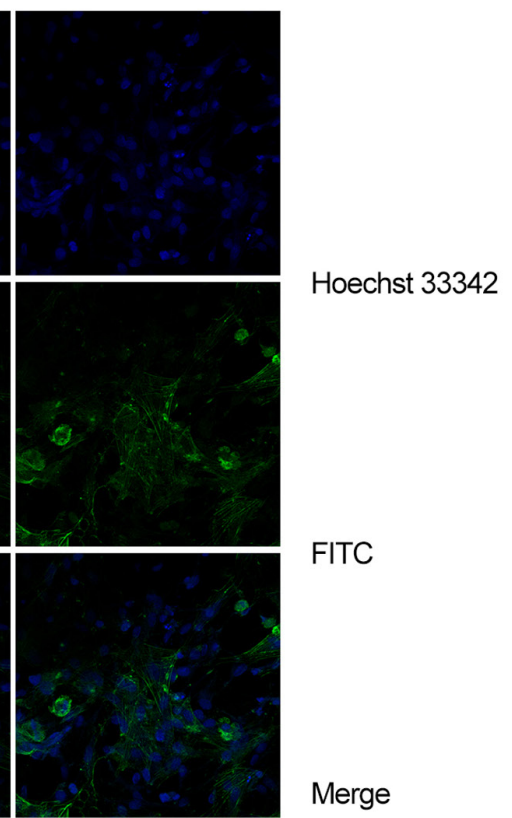

Hoechst 33342

FITC

Merge

Figure 4. Cytoskeletal and nuclear staining of injured podocytes in different groups (magnification, x400). (A) Control group; (B) podocyte injury group; (C) ASTL group; (D) ASTM group; (E) ASTH group. ASTL/M/H, treatment with low/medium/high concentration of astragaloside; FITC, fluorescein isothiocyanate.

AST inhibits ADR-induced decreases of MMP-2 and MMP-9 expression in podocytes. The results of the ELISA indicated that ADR treatment significantly downregulated the protein expression of MMP-2 and MMP-9 $(\mathrm{P}<0.05)$, while the low, medium and high concentrations of AST significantly inhibited these declines in the podocyte injury group $(\mathrm{P}<0.05$; Table II).

Effects of AST on the cytoskeleton of podocytes with $A D R$-induced injury. As presented in Fig. 4, after $24 \mathrm{~h}$ of incubation, the podocyte skeleton in the control group exhibited bright green filaments, arranged in parallel along the cell polarity with the cell microfilament structure being intact and clear. The nuclei had regular and clear shapes with uniform blue staining, and were located in the cell center. However, in the podocytes in the ADR injury group, F-actin was accumulated with irregular shapes, faint color and uneven distribution. The microfilament structure was not intact, the nuclear shape was irregular, the cytoplasm and nuclear areas appeared non-specifically stained and apoptotic bodies and micronuclei were visible. The podocyte microfilaments co-treated with 
Table II. Expression levels of MMP2 and MMP9 proteins in different groups.

\begin{tabular}{lcc}
\hline Group & MMP-2 & MMP-9 \\
\hline Control & $1.00 \pm 0.00$ & $1.00 \pm 0.00$ \\
Podocyte injury & $0.44 \pm 0.05^{\mathrm{a}}$ & $0.36 \pm 0.03^{\mathrm{a}}$ \\
ASTL & $0.56 \pm 0.04^{\mathrm{b}}$ & $0.52 \pm 0.02^{\mathrm{b}}$ \\
ASTM & $0.77 \pm 0.05^{\mathrm{c}}$ & $0.80 \pm 0.03^{\mathrm{c}}$ \\
ASTH & $0.78 \pm 0.07^{\mathrm{c}}$ & $0.73 \pm 0.03^{\mathrm{c}}$ \\
\hline
\end{tabular}

${ }^{\mathrm{a}} \mathrm{P}<0.01$ vs. the control group; ${ }^{\mathrm{b}} \mathrm{P}<0.05,{ }^{\mathrm{c}} \mathrm{P}<0.01$ vs. the podocyte injury group. Values are expressed as the mean \pm standard deviation. MMP, matrix metalloproteinase; ASTL/M/H, treatment with low/medium/high concentration of astragaloside.

a low concentration of AST presented with clearly bundled fibers without polar arrangement characteristics and were loose. However, non-specific staining and apoptotic bodies in the cytoplasm and nuclear areas were still observed. Compared with the control group, the microfilament structure of podocytes co-treated with the medium and high concentration of AST was not clear, but appeared more intact than that in the ADR injury group and the ASTL group.

\section{Discussion}

ADR is an antibiotic that is commonly used as an anti-tumor drug in the clinic. However, the oxidation of semiquinone free radical products formed by its metabolism in the kidneys results in the generation of large amounts of reactive oxygen species, which cause irreversible damage to the glomerular ultrafiltration membrane (15). To investigate this, the ADR-induced podocyte injury model is widely used (16-20), as this method is simple and likely to cause significant typical manifestations of podocyte injury and the changes in cell morphology, viability and podocyte-specific proteins conform to the typical manifestations of human glomerular podocyte injury, which may therefore be used to study this condition. In the present study, the experimental results indicated that ADR significantly reduced the podocyte survival rate and intervention with different concentrations of AST effectively inhibited the reduction of the podocyte survival rate. Therefore, AST protected podocytes from the damaging effect of ADR. However, no dose-dependent effect was observed among the AST treatment groups (ASTL, ASTM and ASTL), where the protective effect of the medium dose AST against podocyte injury was highest.

The podocyte cytoskeleton mainly consists of F-actin, the changes of which may lead to foot process effacement, alter the cell phenotype and induce podocyte apoptosis (21). The results of the laser confocal microscopic analysis performed in the present study indicated that the structural integrity of the nucleus and cytoskeleton of podocytes with ADR-induced injury was severely damaged. It was indicated that the ADR-induced podocyte injury model was successfully established in the present study. However, intervention with the medium and high concentration of AST maintained the structural integrity of the cytoskeleton. In addition, compared with the cell migration ability in the podocyte injury group, the migration rates of podocytes co-treated with the medium and high concentration of AST were significantly higher, which indicated that the podocytes treated by AST retained a better cytoskeletal integrity. While it was demonstrated that treatment with AST had a protective effect against podocyte injury, the precise underlying mechanism remains elusive.

MMPs are a specific group of enzymes that perform extracellular matrix degradation by zinc-dependent protein hydrolysis. It has been indicated that MMPs, particularly MMP-2 and MMP-9, have a key role in podocyte injury (22). Previous studies have also demonstrated that ADR-induced inhibition of podocyte migration and cell injury may be associated with the downregulation of MMP-2 and MMP-9 at the mRNA and protein level $(22,23)$, which was consistent with the results of the present study. Furthermore, the present study indicated that AST increased the expression of MMP-2 and MMP-9 compared with that in the podocyte injury group. It was demonstrated that the protective effect of AST on the proliferation and migration ability of ADR-injured podocytes may be associated with the upregulation of the expression of MMP-2 and MMP-9. In future studies, the molecular signaling of the MMP pathway should be further assessed, which may reveal the underlying mechanisms.

Oxidative stress has a key role in the pathogenesis of various diseases. Antioxidants protect cells and tissues from oxidative stress by scavenging free radicals and reactive oxygen species. The reactive oxygen species generated during oxidative stress-associated processes reduce the expression of $\alpha 3 \beta 1$, promote lipid peroxidation and affect cellular signaling cascades, thereby damaging podocytes (20). In the present study, the cell damage index LDH and the oxidative stress parameter MDA were significantly increased, while the activity of SOD, an antioxidant index, was significantly decreased in ADR-induced podocytes compared with those in the normal control group. However, AST decreased LDH and MDA levels and increased SOD activity compared with those in the podocyte injury group, and the medium concentration of AST was most effective. Glomerular podocytes are a highly differentiated end-stage cell type, whose proliferation ability is limited and they are difficult to regenerate once damaged. In the present study, intervention with AST was likely associated with the reduction of the oxidative stress response of podocytes.

In conclusion, co-treatment with AST maintained a balance of the oxidative stress environment in MPC5 podocytes with ADR injury to improve their migration ability and inhibit the rearrangement and destruction of the cytoskeleton. As a possible mechanism, AST inhibits ADR-induced decreases in the expression of MMP-2, MMP-9 in order to protect against podocyte injury.

\section{Acknowledgements}

Not applicable.

\section{Funding}

This study was funded by the Gansu Provincial Administration of Traditional Chinese Medicine Research 
(Gansu Province Administration of Traditional Chinese Medicine, grant no. G2K-2014-59).

\section{Availability of data and materials}

The analyzed data sets generated during the study are available from the corresponding author on reasonable request.

\section{Authors' contributions}

YPS and YCS designed the present study, analyzed the data and wrote the manuscript; XXC and XL established the podocyte injury model; JL and WJC assisted with technical performance and contributed to writing the manuscript. All authors read and approved the final manuscript. YPS and YCS contributed equally to the present study as first authors. The final version of the manuscript has been read and approved by all authors and each author believes that the manuscript represents honest work.

\section{Ethical approval and consent to participate}

Not applicable.

\section{Consent for publication}

Not applicable.

\section{Competing interests}

The authors declare that they have no competing interests.

\section{References}

1. Pavenstadt H, Kriz W and Kretzler M: Cell biology of the glomerular podocyte. Physiol Rev 83: 253-307, 2003.

2. Shankland SJ and Al' Douahji M: Cell cycle regulatory proteins in glomerular disease. Exp Nephrol 7: 207-211, 1999.

3. Zhu C, Xuan X, Che R, Ding G, Zhao M, Bai M, Jia Z, Huang S and Zhang A: Dysfunction of the PGC- $1 \alpha$-mitochondria axis confers adriamycin-induced podocyte injury. Am J Physiol Renal Physiol 306: F1410-F1417, 2014.

4. Maezawa Y, Onay T, Scott RP, Keir LS, Dimke H, Li C, Eremina V, Maezawa Y, Jeansson M, Shan J, et al: Loss of the podocyte expressed transcription factor Tcf $21 / \operatorname{Pod} 1$ results in podocyte differentiation defects and FSGS. J Am Soc Nephrol 25: 2459-2470, 2014.

5. Hanamura K, Tojo A and Fujita T: Urinary and glomerular podocytes in patients with chronic kidney diseases. Clin Exp Nephrol 18: 95-103, 2014.

6. Takeda A, Ohgushi H, Niimura F and Matsutani H: Long-term effects of immunosuppressants in steroid-dependent nephrotic syndrome. Pediatr Nephrol 12: 746-750, 1998.
7. Hogan J and Radhakrishnan J: The treatment of minimal change disease in adults. J Am Soc Nephrol 24: 702-711, 2013.

8. Shahzad M, Shabbir A, Wojcikowski K, Wohlmuth H and Gobe GC: The Antioxidant effects of radix astragali (astragalus membranaceus and related species) in protecting tissues from injury and disease. Curr Drug Targets 17: 1331-1340, 2016.

9. Liu G, Song J, Guo Y, Wang T and Zhou Z: Astragalus injection protects cerebral ischemic injury by inhibiting neuronal apoptosis and the expression of JNK3 after cerebral ischemic reperfusion in rats. Behav Brain Funct 9: 36, 2013.

10. Feng M, Yuan W, Zhang R, Fu P and Wu T: Chinese herbal medicine Huangqi type formulations for nephrotic syndrome. Cochrane Database Syst Rev 6: Cd006335, 2013.

11. Korbet SM: Treatment of primary FSGS in adults. J Am Soc Nephrol 23: 1769-1776, 2012.

12. Yuan W, Wang J and Wu T: Chinese herbal medicine Huangqi type formulations for nephrotic syndrome. Cochrane Database Syst Rev 2: Cd006335, 2008.

13. Xu M, Yin J, Xie L, Zhang J, Zou C, Zou J, Liu F, Ju W and Li P: Pharmacokinetics and tolerance of toal astragalosides after intravenous infusion of astragalosides injection in healthy Chinese volunteers. Phytomedicine 20: 1105-1111, 2013.

14. Wang N, Wei RB, Li QP, Yang X and Chen X: Protective effects of astragaloside in rats with adriamycin nephropathy and underlying mechanism. Chin J Nat Med 14: 270-277, 2016.

15. Bertani T, Poggi A, Pozzoni R, Delaini F, Sacchi G, Thoua Y, Mecca G, Remuzzi G and Donati MB: Adriamycin-induced nephrotic syndrome in rats: Sequence of pathologic events. Lab Invest 46: 16-23, 1982.

16. Yi M, Zhang L, Liu Y, Livingston MJ, Chen JK, Nahman NS Jr, Liu F and Dong Z: Autophagy is activated to protect against podocyte injury in adriamycin-induced nephropathy. Am J Physiol Renal Physiol 313: F74-F84, 2017.

17. Saito Y, Okamura M, Nakajima S, Hayakawa K, Huang T, Yao J and Kitamura M: Suppression of nephrin expression by TNF-alpha via interfering with the cAMP-retinoic acid receptor pathway. Am J Physiol Renal Physiol 298: 1436-1444, 2010.

18. Zhang HT, Wang WW, Ren LH, Zhao XX, Wang ZH, Zhuang DL and Bai YN: The mTORC2/Akt/NFKB pathway-mediated activation of TRPC6 participates in adriamycin-induced podocyte apoptosis. Cell Physiol Biochem 40: 1079-1093, 2016.

19. Wang N, Wei RB, Li P, Li QP, Yang X, Yang Y, Huang MJ, Wang R, Yin Z, Lv Y and Chen XM: Treatment with irbesatan may improve slit diaphragm alterations in rats with adriamycin-inducednephropathy. J Renin Angiotensin Aldosterone Syst 17: 1470320316646884, 2016.

20. Susztak K, Raff AC, Schiffer M and Böttinger EP: Glucose-induced reactive oxygen species cause apoptosis of podocytes and podocyte depletion at the onset of diabetic nephropathy. Diabetes 55: 225-233, 2006.

21. Asanuma K, Yanagida-Asanuma E, Faul C, Tomino Y, Kim K and Mundel P: Synaptopodin orchestrates actin organization and cell motility via regulation of RhoA signaling. Nat Cell Biol 8: 485-491, 2006

22. Lei FY, Zhou TB, Qin YH, Chen XP and Li ZY: Potential signal pathway of all-trans retinoic acid for MMP-2 and MMP-9 expression in injury podocyte induced by adriamycin. J Recept Signal Transduct Res 34: 378-385, 2014

23. Merkle M, Ribeiro A, Köppel S and Wörnle M: TNF- $\alpha$ enhances TLR3-dependent effects on MMP-9 expression in human mesangial cells. Cell Biol Int 36: 1155-1160, 2012. 